\title{
Evidence for an association of gut microbial Clostridia with brain functional connectivity and gastrointestinal sensorimotor function in patients with irritable bowel syndrome, based on tripartite network analysis
}

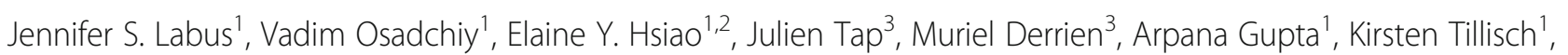
Boris Le Nevé, Cecilia Grinsvall ${ }^{4}$, Maria Ljungberg ${ }^{5}$, Lena Öhman ${ }^{4,6}$, Hans Törnblom ${ }^{4}$, Magnus Simren ${ }^{4,7 \dagger}$ and Emeran A. Mayer ${ }^{1 * \dagger}$

\begin{abstract}
Background and aims: Evidence from preclinical and clinical studies suggests that interactions among the brain, gut, and microbiota may affect the pathophysiology of irritable bowel syndrome (IBS). As disruptions in central and peripheral serotonergic signaling pathways have been found in patients with IBS, we explored the hypothesis that the abundance of serotonin-modulating microbes of the order Clostridiales is associated with functional connectivity of somatosensory brain regions and gastrointestinal (GI) sensorimotor function.
\end{abstract}

Methods: We performed a prospective study of 65 patients with IBS and 21 healthy individuals (controls) recruited from 2011 through 2013 at a secondary/tertiary care outpatient clinic in Sweden. Study participants underwent functional brain imaging, rectal balloon distension, a nutrient and lactulose challenge test, and assessment of oroanal transit time within a month. They also submitted stool samples, which were analyzed by $16 \mathrm{~S}$ ribosomal RNA gene sequencing. A tripartite network analysis based on graph theory was used to investigate the interactions among bacteria in the order Clostridiales, connectivity of brain regions in the somatosensory network, and $\mathrm{Gl}$ sensorimotor function.

Results: We found associations between Gl sensorimotor function and gut microbes in stool samples from controls, but not in samples from IBS patients. The largest differences between controls and patients with IBS were observed in the Lachnospiraceae incertae sedis, Clostridium XIVa, and Coprococcus subnetworks. We found connectivity of subcortical (thalamus, caudate, and putamen) and cortical (primary and secondary somatosensory cortices) regions to be involved in mediating interactions among these networks.

(Continued on next page)

\footnotetext{
* Correspondence: emayer@ucla.edu

${ }^{\dagger}$ Magnus Simren and Emeran A. Mayer share senior authorship

${ }^{1} \mathrm{G}$. Oppenheimer Center for Neurobiology of Stress \& Resilience, UCLA

Vatche and Tamar Manoukian Division of Digestive Diseases, UCLA CHS

42-210, MC737818, 10833 Le Conte Avenue, Los Angeles, CA 90095-7378,

USA

Full list of author information is available at the end of the article
}

(c) The Author(s). 2019 Open Access This article is distributed under the terms of the Creative Commons Attribution 4.0 International License (http://creativecommons.org/licenses/by/4.0/), which permits unrestricted use, distribution, and reproduction in any medium, provided you give appropriate credit to the original author(s) and the source, provide a link to the Creative Commons license, and indicate if changes were made. The Creative Commons Public Domain Dedication waiver (http://creativecommons.org/publicdomain/zero/1.0/) applies to the data made available in this article, unless otherwise stated. 


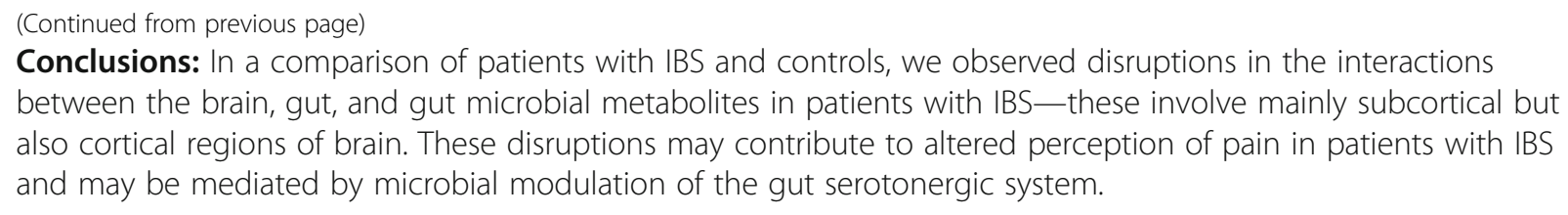

Keywords: Central nervous system, Brain-gut axis, Brain imaging, Microbiome, Bacteria,

\section{Introduction}

Irritable bowel syndrome (IBS) is a common disorder characterized by chronically recurring abdominal pain associated with altered bowel habits [1]. Even though alterations in the brain-gut-microbiome axis have been implicated as an important component, IBS pathophysiology and the role of altered gut microbiome-brain interactions are incompletely understood [1]. Previous brain imaging studies have identified functional and structural differences within the brain, including in regions of the sensorimotor, salience, and emotion regulating networks between healthy controls ( $\mathrm{HCs})$ and IBS patients [2,3].

Visceral hypersensitivity and gastrointestinal (GI) motor function abnormalities are considered to be of primary importance for IBS symptom generation. Balloon distension in different parts of the GI tract, during which patients report the extent of pain and discomfort experienced, has been used to assess enhanced perception of visceral stimuli ("evoked visceral hypersensitivity") in patients with functional GI disorders. A quantitative meta-analysis focused on changes in brain activation during rectal distension identified greater engagement of regions associated with endogenous pain processing and modulation, such as the basal ganglia, in IBS compared to HCs [4]. Studies involving large cohorts of IBS subjects have shown modest associations between GI symptom severity and evoked visceral sensitivity, assessed through balloon distension $[5,6]$, as well as between IBS symptoms and abnormal GI motor function [7]. These modest associations may reflect a role for other aspects of brain-gut signaling, that may play a similar or greater role in symptom generation.

Reports of alterations in the gut microbiome in patients meeting IBS symptom criteria have been inconsistent, likely due to heterogeneity of gut microbial composition in IBS, differences in sampling and analysis protocols, and a lack of information about the causality of brain gut microbiome interactions in symptom generation $[8,9]$. Despite these inconsistent findings, several pieces of evidence suggest a role of Clostridiales-associated species in IBS. In a previous study, we have demonstrated that enterotypes composed of Clostridiales-associated species comprise a multivariate microbial signature that associates with IBS symptom severity [10]. In another study, we showed that the gut microbial composition of IBS patients shows an increase in members of the class Clostridia, which correlates with volumes of brain regions in the sensorimotor network [11]. Importantly, recent evidence obtained in mouse models reveals an important role for colonic spore-forming bacteria in the order Clostridiales (particularly enriched with members of families Ruminococcaceae and family Lachnospiraceae) in stimulating the biosynthesis and release of serotonin (5-HT) from intestinal enterochromaffin cells and modulating GI motility [12]. Dysfunctions of the serotonergic system have been extensively studied within the context of IBS due to the prominent role of 5-HT in secretion, absorption, and intestinal transit in the GI tract, and mood, pain modulation, and cognitive function within the central nervous system (CNS) [13]. Therapeutic agents targeting 5-HT signaling, including direct 5-HT receptor modulators and 5-HT selective reuptake inhibitors, have been investigated as treatment options, achieving moderate efficacy in certain patients with IBS [14]. Additionally, several studies have demonstrated correlations between 5-HT plasma levels and post-prandial symptomology and sigmoid colon activity $[13,15,16]$.

Although the mechanisms driving gut microbiota-initiated signaling to the brain in humans are not known, this interaction may be mediated by the microbial production of small-molecules, which can signal to the brain via afferent vagal fibers, the immune system, or directly through the circulation. Examples include short-chain fatty acids or secondary bile acids, which have been documented to interact with specific receptors on enterochromaffin cells, thereby modulating the gut serotonergic system [12]. Based on the close physical connections between enterochromaffin cells and vagal afferent nerve terminals, such modulation could lead to 5-HT-mediated vagal afferent signaling to the medullary nucleus tractus solitarius and to higher emotional and autonomic networks in the brain. The vagus nerve is a key component in the microbiota-gut-brain axis interface [17] and a major pathway providing the brain with interoceptive as well as microbial information from the gut. Further support for microbial generated metabolites in IBS symptom generation include the beneficial effects of diets that eliminate fermentable oligosaccharides, disaccharides, monosaccharides, and polyols (FODMAP) [18]. FODMAPs are short-chain carbohydrates that are poorly absorbed and rapidly fermented by 
gut bacteria to produce metabolites that can influence the gut microbiota, gut barrier, immune response, and visceral sensation [19]. In addition to their role in gut brain signaling, microbial-derived metabolites may directly activate the enteric nervous system, modulating contractile activity and secretion [20].

In addition to identifying functional alterations in specific brain regions, more recent efforts have focused on identifying alterations in the architecture and connectivity of brain networks [21]. Brain connectivity can be assessed using structural and functional network analysis via graph theory. Within this framework, brain regions are characterized by measures that quantify their contribution to the functional and anatomical integrity and information flow in the whole brain network [22-25].

In this exploratory study, we build on the considerable evidence implicating certain gut microbes (order Clostridiales) and regions of the sensorimotor network in IBS. We use a biological system-based, data-driven approach to interrogate and visualize the relationship between relative abundance of several species in the order Clostridiales (families Ruminococcaceae and Lachnospiraceae) with GI sensorimotor function and connectivity of key regions of the brain's sensorimotor network in HC and IBS patients. Even though findings from this exploratory study will need to be validated in a much larger sample, they provide initial support for role of alterations in the brain-gut-microbiome axis in symptom generation in IBS.

\section{Methods}

\section{Subjects}

Adult IBS patients $(n=65)$, aged $18-65$ years, were recruited between 2011 and 2013 at a secondary/tertiary care outpatient clinic (Sahlgrenska University Hospital, Gothenburg, Sweden). As subjects were recruited in 2011-2013, IBS was diagnosed based on the Rome III criteria [26]. A gastroenterologist (H.T. or M.S.) diagnosed the patients. Exclusion criteria included (1) the use of probiotics or antibiotics during the study period or within 1 month before inclusion; (2) another diagnosis that could explain the GI symptoms such as celiac disease, inflammatory bowel disease, or microscopic colitis; (3) severe psychiatric disease as the dominant clinical problem; (4) any other severe diseases; and (5) a history of drug or alcohol abuse. This study did not focus on only one subgroup of IBS as previous studies $[10,11,27]$ have shown only modest associations between IBS subtype defined by symptom criteria, gut microbiota composition, and brain imaging differences. A HC group $(n=21)$ was recruited by use of advertisement and checked by interview and questionnaire to exclude chronic diseases and any current GI symptoms. All subjects were of Caucasian origin. All subjects completed a questionnaire to characterize their symptom severity using the IBS Severity Scoring system (IBS-SSS), and according to validated cut-off levels, the patients were categorized as having mild IBS (score of < 175), moderate IBS (175-300), or severe IBS $(>300)$ [28]. Even though the IBS-SSS has not been validated in healthy subjects, we use it here as a proxy for the assessment of mild abdominal symptoms, not meeting IBS diagnostic criteria. Data from these subjects has been previously published as a part of a larger study [10]. Here, we analyze a subset of individuals, 65 IBS patients (46 females) and $21 \mathrm{HCs}$ (16 females), all of whom underwent functional brain imaging. Among $\mathrm{HCs}$, none of the subjects were taking any medications. Among IBS patients, seven subjects (11\%) were taking a proton-pump inhibitor and ten subjects were taking a daily psychotropic medication. As mentioned above, these medications were discontinued $48 \mathrm{~h}$ prior to investigation.

All investigations were performed during a 1-month period, and in the same order for each patient with oroanal transit time (OATT) and rectal sensitivity testing performed during the same day, MRI 7-10 days after that, stool sample collection 3-5 days thereafter, and the lactulose challenge test at the end of this month.

All subjects were given verbal and written information about the study prior to providing written informed consent to participate. The Regional Ethical Review Board in Gothenburg approved the study before the start of subject inclusion.

\section{Measurement of $\mathrm{Gl}$ sensorimotor function Evoked visceral sensitivity: rectal barostat procedures}

After an overnight fast and a $500-800 \mathrm{~mL}$ tap water enema, a balloon catheter was inserted into the rectum, so that the middle of the bag was located approximately $10 \mathrm{~cm}$ from the anal verge, and connected to a computer-driven electronic barostat (Dual Drive Barostat, Distender Series II; G\&J Electronics Inc., Toronto, ON, Canada). After a 20-30-min recovery period, the pressure in the balloon was increased from $4 \mathrm{mmHg}$ in steps of $1 \mathrm{mmHg}$ for $1 \mathrm{~min}$ per step until respiratory excursions were observed. The baseline operating pressure (BOP) was defined as $2 \mathrm{mmHg}$ above the minimal distension pressure at which respiratory excursions were clearly recorded from the barostat tracing. Next, an initial conditioning distension sequence was performed in which the pressure was increased from 0 to $20 \mathrm{mmHg}$ in steps of $4 \mathrm{mmHg}$ for $15 \mathrm{~s}$ per step. Thereafter, an ascending method of limits rectal distension protocol was used, with ramp inflation starting at $0 \mathrm{mmHg}$ increasing by steps of $4 \mathrm{mmHg}$ with $1 \mathrm{~min}$ per step to identify sensory thresholds. For this study, we used rectal pain and discomfort thresholds, in addition to first rectal sensation. 
The distension sequence progressed until pain was reported or until the maximal distension of $60 \mathrm{mmHg}$ was achieved [29].

During the second half of the procedure, subjects randomly received fixed phasic distensions at 12, 24, 36, and $48 \mathrm{mmHg}$ above the BOP and were asked to complete visual analogue scale ratings for rectal sensations after $30 \mathrm{~s}$ of distension. The distension lasted for $60 \mathrm{~s}$ with an inter-stimulus interval of $2 \mathrm{~min}$. During the phasic distension, only one distension level above the pain threshold was delivered for ethical reasons. Most participants completed the 12 and $24 \mathrm{mmHg}$ distension levels, but fewer continued to 36 and $48 \mathrm{mmHg}$ distension. For this reason, we included visual analogue scale ratings for the $24 \mathrm{mmHg}$ phasic distension in our analyses, and only pain and discomfort rating were used in this study (rectal pain and discomfort intensity, respectively).

\section{OATT}

OATT using a radiopaque marker study [7] was determined in all subjects. Any medications with known effects on the GI tract (proton pump inhibitors, laxatives, antidiarrheals, opioid analgesics, prokinetics, spasmolytics, antidepressants) were discontinued at least $48 \mathrm{~h}$ before intake of the first radiopaque markers. The subjects ingested ten radiopaque markers each day for 6 days. On the morning of the 7th day, the radiopaque rings still present in the bowel were counted upon arrival at the laboratory, using fluoroscopy (Exposcop 7000 Compact, Ziehm GmbH, Nüremberg, Germany). OATT expressed in days was calculated by dividing the number of retained radiopaque markers by the daily dose number; i.e., 10 .

\section{Nutrient and lactulose challenge test}

This challenge has been previously described in detail [30]. Briefly, all participants arrived to the laboratory at 7:30 AM after an overnight fast. The combined nutrient and lactulose challenge test $(400 \mathrm{~mL}$ Nutridrink (Danone Nutricia, Utrecht, The Netherlands), $1.5 \mathrm{kcal} /$ $\mathrm{mL}, 16 \%$ protein, $49 \%$ carbohydrate, $35 \%$ fat, gluten free, lactose $<0.025 \mathrm{~g} / 100 \mathrm{~mL}, 25 \mathrm{~g}$ lactulose) was served at 8:00 AM. The severity of 8 GI symptoms and the overall digestive comfort were assessed every $15 \mathrm{~min}$ starting $30 \mathrm{~min}$ before the test meal and during $4 \mathrm{~h}$ after meal intake. In this study, we only included the $4 \mathrm{~h}$ mean area under the curves for the pain and discomfort ratings during the challenge test (lactulose challenge-pain and discomfort, respectively).

Fecal sample DNA extraction and microbial composition assessment

Fecal samples were collected in RNAlater solution (Ambion, Courtaboeuf, France) at subjects' homes, stored at room temperature, and were returned to the laboratory within 3-4 days in the majority of cases. In a small number of cases, samples were returned within 3 weeks. DNA was extracted using mechanical lysis (Fastprep FP120; ThermoSavant, Illkirch, France) followed by phenol/chloroform-based extraction as previously described [31]. Hypervariable 16S ribosomal RNA (rRNA) regions (V5-V6) were amplified using primers 5'-AGGATTAGATACCCTGGTA-3' and 5'-CRRCACG AGCTGACGAC-3'. DNA Vision SA (Charleroi, Belgium) performed sequencing using a 454 Life Sciences Genome Sequencer FLX instrument (Roche, Basel, Switzerland). Raw reads quality filtering and trimming, operational taxonomic units (OTU) clustering, and taxonomic assignment were performed using the LotuS v1.32 pipeline [32] as previously described [10].

\section{Resting state neuroimaging}

T1-weighted images were acquired on a 3 Tesla Philips Achieva using the standard 8-channel head coil, with a T1-weighted 3D TFE gradient echo scan, i.e., a magnetization-prepared rapid acquisition gradient echo $($ MP-RAGE $)$ sequence, repetition time $=7.0 \mathrm{~ms}$, echo time $=3.2 \mathrm{~s}$, inversion time $=900 \mathrm{~ms}$, flip angle $=9^{\circ}$, field of view $=256$ by $220 \mathrm{~mm}$, slices per volume $=176$, slice thickness $=1 \mathrm{~mm}$, voxel size $=1 \times 1 \times 1 \mathrm{~mm}$. Resting state fMRI (eyes closed) was acquired using Philips SENSE parallel imaging with a reduction factor $=2$, repetition time $=2000 \mathrm{~ms}$, echo time $=30 \mathrm{~ms}$, flip angle $=77^{\circ}$, field of view $250 \times 250 \mathrm{~mm}$, acquisition matrix $=$ $72 \times 72$, slice thickness $=4 \mathrm{~mm}$, reconstructed voxel size $=4 \times 2.6 \times 2.6 \mathrm{~mm}$. In preparations before the scan, the subjects were asked not to take any analgesics, anti-histamines, alcohol, or nicotine for a minimal of $12 \mathrm{~h}$ before the scan; not to take any psychopharmacological drugs for $48 \mathrm{~h}$ before the scan; not to ingest caffeine for 2-3 $\mathrm{h}$ before the scan; and to avoid strenuous physical activity on the day before the scan.

\section{Quality control}

Preprocessing for quality control involved bias field correction, coregistration, motion correction, spatial normalization, tissue segmentation, and Fourier transformation for frequency distribution were performed at the Bioinformatics and Neuroimaging core of the G. Oppenheimer Center for Neurobiology of Stress and Resilience at UCLA. Functional images were included based on compliance with acquisition protocol, full brain coverage, motion estimate of $<1 / 2$ voxel size between adjacent time points, low standard deviation across time series for all voxels, ghosting in cerebrum, minimal physiological noise $(>0.2 \mathrm{~Hz}$ in frequency spectrum), and few to no outlier voxels, mean intensity shifts, or K-space "spikes." 


\section{Brain Parcellation}

Segmentation and regional parcellation of gray matter images was performed using FreeSurfer (Dale, Fischl et al. 1999; Fischl, Sereno et al. 1999; Fischl, Salat et al. 2002) and workflow pipelines at UCLA developed in collaboration with the UCLA Laboratory of Neuroimaging pipeline using the Destrieux atlas and the Harvard-Oxford atlas [24]. This parcellation yielded 74 cortical structures, 7 subcortical structures, the cerebellum, and the brainstem, for a complete set of 165 parcellations of the entire brain.

\section{Functional brain network construction}

The parcellation and the functional connectivity results were combined to produce a $165 \times 165$ weighted, undirected connectivity matrix. Resting-state image preprocessing was performed using the SPM8 software (Wellcome Department of Cognitive Neurology, London, UK). Images were transformed from DICOM into NIfTI, slice-time corrected, co-registered with the high-resolution structural images, spatially normalized to the MNI space, and resampled to a voxel size of $2 \times$ $2 \times 2 \mathrm{~mm}$. Normalized functional images were further preprocessed and analyzed using the SPM-based CONN toolbox version 13 (www.nitrc.org/projects/conn). The resting-state images were filtered using a band-pass filter $(0.008 / \mathrm{s}<f<0.08 / \mathrm{s})$ to reduce the low- and high-frequency noises. A component based noise-correction method, CompCor [33], was applied to remove nuisances for better sensitivity and specificity of the analysis. Six motion realignment parameters and confounds for white matter and CSF were removed using regression. Region-to-region functional connectivity, cross-correlations between the blood oxygenated level-dependent time series, were computed between all the brain regions in CONN toolbox. The connectivity correlation coefficients were then used to construct the final functional network setting negative values to zero. The magnitude of the correlation represents the weights of the specific region in the functional network.

\section{Computing network metrics}

The Graph Theoretic GLM tool (www.nitrc.org/projects/ metalab_gtg) [34] and in-house matlab workflow scripts were used to calculate and analyze the functional brain network properties and organization from the subject-specific functional brain networks. Several regional weighted network metrics indexing centrality were computed. Regions with high centrality are highly influential within a network, communicate with many other regions, facilitate functional integration, and play a key role in network resilience to insult [25]. Three indices of centrality were computed: (1) Degree strength reflects the number of other regions a brain region interacts with functionally (local prominence), (2) betweenness centrality, reflecting the ability of a region to influence information flow (signaling) between two other regions, and (3) eigenvector centrality, where higher values indicate the region is directly connected to other highly connected regions reflective of the global (vs. local) prominence of a region.

\section{Selection of microbial genera}

In order to increase statistical power, we selected genera based on previous work, which showed that spore formers particularly enriched with members of order Clostridiales, families Ruminococcaceae and Lachnospiraceae, stimulate the biosynthesis and release of 5-HT from intestinal enterochromaffin cells and modulate GI motility $[11,12]$. Only genera in these families were included in our analysis. Any genus with greater than $80 \%$ missing data in either the IBS or HC group was excluded. The genera that fulfilled these criteria and were included in this study are families Ruminococcaceae (genera: Clostridium IV, Faecalibacterium, and Oscillibacter) and Lachnospiraceae (genera: Clostridium XIVa, Clostridium $X I V b$, Blautia, Coprococcus, Roseburia, and Lachnospiracea incertae sedis).

\section{Statistical analysis}

Tripartite network analysis was performed to integrate information from three data sets: (1) abundance of bacterial genera (relative to all other genera) in the order Clostridiales including families Ruminococcaceae and Lachnospiraceae, (2) GI sensorimotor function data, including IBS-SSS, and (3) the functional network metrics characterizing regions of interest (Table 1), including mid- and posterior insula ( $\mathrm{m}$ and pINS), somatosensory cortex, basal ganglia (nucleus accumbens [NAcc], caudate nucleus, putamen, and pallidum), and thalamus. For reasons similar to the restriction of microbial taxa, the selection of brain regions of a sensorimotor network was based on previous structural and resting state brain imaging studies in IBS consistently demonstrating alterations in the regions of the sensorimotor network, with a recent study showing associations between gut microbial abundance and differences in sensorimotor regions [11, 35-37] (Additional file 1: Table S1 and Additional file 2: Table S2).

The interaction between the phenome (GI sensorimotor function and IBS symptoms), microbiome (stool microbial community), and connectome (indices of regional centrality in the brain) was determined by computing Spearman's correlations between different data types controlling for age and sex in Matlab version R2015b. An effect size of $r=0.50$ is considered large (25\% variance explained), 0.30 medium (9\% variance explained), and 0.10 small (1\% variance explained) [38]. 
Table 1 Regions of Interest from the Destrieux and Harvard-Oxford Atlas

\begin{tabular}{|c|c|c|}
\hline Region & Description & Abbreviation \\
\hline \multirow[t]{3}{*}{ Primary somatosensory cortex (S1) } & Postcentral gyrus & PosCG \\
\hline & Postcentral sulcus & PosCS \\
\hline & Central sulcus & CS \\
\hline Secondary somatosensory cortex (S2) & Subcentral gyrus (central operculum) and sulcus & SbCG_S \\
\hline \multirow[t]{3}{*}{ Primary motor cortex (M1) } & Precentral gyrus & PRCG \\
\hline & Inferior part of the precentral sulcus & InfPrCS \\
\hline & Superior part of the precentral sulcus & SupPrCs \\
\hline \multirow[t]{2}{*}{ Supplementary motor area (M2/SMA) } & Superior frontal gyrus & SupFG \\
\hline & Superior frontal sulcus & SupFS \\
\hline Anterior/middle insula (a/mINS) & Superior segment of the circular sulcus of the insula & SupCirlnS \\
\hline \multirow[t]{2}{*}{ Middle/posterior insula (m/pINS) } & Long insular gyrus and central sulcus of the insula & LolnG_CInS \\
\hline & Inferior segment of the circular sulcus of the insula & InfCirlnS \\
\hline Posterior insula (pINS) & Posterior ramus of the lateral sulcus & PosLS \\
\hline \multirow[t]{4}{*}{ Basal ganglia } & Putamen & $\mathrm{Pu}$ \\
\hline & Caudate nucleus & $\mathrm{CaN}$ \\
\hline & Nucleus accumbens & NAcc \\
\hline & Globus pallidus & Pal \\
\hline Thalamus & Thalamus & Tha \\
\hline
\end{tabular}

Fisher's r-to-z transformation was applied to evaluate the correlation coefficient difference between groups (IBS-HC), using the $Z$ test. Significance was considered at uncorrected $p<0.05$. Parameter estimates and significance values from the group comparison are provided in Additional file 3: Table S3. No corrections for multiple testing were employed as our goal for this exploratory research was to generate a number of hypotheses for further testing and confirmation in a larger sample. Using $G^{*}$ Power, we perform a post-hoc analysis to determine the effect size, $r$, our samples could detect with adequate power $(80 \%)$ based on an alpha $=0.05$ (uncorrected $p$ ), and a two-tailed test for a significant correlation. For the IBS sample $(N=65)$, we only had adequate power to detect a significant correlation of $r \geq$ 0.33 , if it existed. For the HC sample $(N=21)$, we only had adequate power for $r \geq 0.54$. We provide further details regarding cohort sizes needed to justify tighter error control in Additional file 4.

To visualize and construct brain, GI sensorimotor function, and stool microbial community interaction networks, Cytoscape v. 3.5.1 was used. First, the difference network (and subnetworks), representing group differences at $p<$ 0.05 , were constructed using the unweighted force-directed layout. This layout uses a physics algorithm to simulate the network as a physical system, where edges attract and nodes repel. This technique organizes the network to avoid crossing edges and overlapping nodes. As such, nodes that are connected and have similar associations are grouped together, allowing for clusters or patterns in the data to emerge. Associations for this network were included if the Pearson's correlations for the IBS and/or the HCs data achieved significance. For genera-specific subnetworks, we select the genus of interest and its first neighbors as nodes and all adjacent edges. The architecture of IBS and HCs networks and subnetworks were based on the significant difference network. Edges (lines) represent significant $Z$ values from the group test and nodes represent data points. Positive $Z$ values were colored red and indicate IBS $>\mathrm{HC}$ and a negative $Z$ value indicates IBS $<\mathrm{HC}$. Individual edge estimates by group are reported in Additional file 1: Table S1 and Additional file 2: Table S2. The results are described in terms of direct (correlations with genera) and indirect effects (GI sensorimotor function and brain connectivity metric that are a part of the interaction network, but not directly correlated with the genus of interest) with a focus on the interaction between genus and GI sensorimotor function. As all subnetworks are derived from significant data identified in the difference network, the analysis presumes that associations present in one group that are missing in another (1) differentiate the groups, and (2) indicate potential clues to the functionality of the system, permitting hypothesis generation for future research. Independent sample $t$ tests were used to test for group differences in clinical variables.

\section{Results}

Clinical and behavioral characteristics

Means, standard deviations, and group comparisons for age, symptom severity, and GI sensorimotor function 
variables are presented in Table 2. No differences in age were noted. As expected, IBS subjects reported significantly higher discomfort and pain ratings during the $24 \mathrm{mmHg}$ rectal balloon distension and during the nutrient and lactulose challenge test. In contrast, no statistically significant differences between the groups were observed for rectal discomfort or pain thresholds, and OATT. On the IBS-SSS, 12 patients were classified as mild, 24 moderate, and 29 severe symptoms. All HCs were classified as mild/no symptoms.

\section{Tripartite network analysis of gut microbe-brain connectivity}

The global tripartite microbial-brain-evoked symptom networks are depicted in Fig. 1a (HC), b (IBS), and c (difference network). These networks show statistical associations between brain, microbial, and GI sensorimotor function parameters. Additional file 1: Table S1, Additional file 2: Table S2, and Additional file 3: Table S3 outline all of the associations for HCs, IBS, and difference networks, respectively, in greater detail. In the following, we describe the results with respect to microbial subnetworks related to four genera within the order Clostridiales: L. incertae sedis, Coprococcus, and Clostridium XIVa and b.

\section{Lachnospiracea incertae sedis}

The HCs subnetwork showed a robust positive association network between Lachnospiraceae incertae sedis and S1, in addition to an indirect association between this genus with rectal pain threshold through connectivity of S1 (Fig. 2a). In contrast to the $\mathrm{HC}$ association network, the IBS subnetwork demonstrated no associations between Lachnospiraceae incertae sedis and pain threshold, but did show a single positive association between this genus and connectivity of S2 (Fig. 2b). The Lachnospiraceae incertae sedis difference network underscores that the observed differences are statistically significant (Fig. 2c). Clostridium XIVa was also a part of the Lachnospiraceae incertae sedis subnetwork.

\section{Coprococcus}

The HC subnetwork showed direct negative associations between Coprococcus and discomfort and pain during the nutrient and lactulose challenge test, in addition to indirect association between this genus and pain reported during this challenge test through connectivity of the caudate (Fig. 3a). In contrast, the IBS subnetwork did not show any significant associations involving Coprococcus (Fig. 3b). The Coprococcus difference network underscores that the observed differences are statistically significant (Fig. 3c). Clostridium XIVa and Clostridium IV were also a part of the Coprococcus subnetwork.

\section{Clostridium XIVa}

The HC subnetwork showed indirect associations between Clostridium XIVa and several measures of visceral sensitivity, including rectal discomfort threshold (through connectivity of the putamen), rectal discomfort intensity (through connectivity of S1), rectal pain threshold (through connectivity of the putamen, NAcc, and S1), and pain during the nutrient and lactulose challenge test (through connectivity of S1). Indirect associations were also observed for OATT (through connectivity of the putamen and NAcc) (Fig. 4a). In contrast, the IBS subnetwork did not demonstrate any associations between Clostridium XIVa and any measures of GI sensorimotor function. However, the IBS subnetwork did show exclusively positive associations between Clostridium XIVa and connectivity of subcortical regions (putamen, caudate, and thalamus) (Fig. 4b). The Clostridium $X I V a$ difference network underscores that the observed differences are significant (Fig. 4c). Coprococcus and Lachnospiraceae incertae sedis were also a part of the Clostridium XIVa subnetwork.

\section{Clostridium XIVb}

The HCs subnetwork showed an indirect association between Clostridium XIVb and IBS-SSS through connectivity of S1 and M1. Additionally, Clostridium IV, which

Table 2 Subject characteristics and Gl sensorimotor function measures

\begin{tabular}{|c|c|c|c|c|c|c|}
\hline & \multicolumn{2}{|c|}{ Healthy controls $(N=21, F=16)$} & \multicolumn{2}{|c|}{$\operatorname{IBS}(N=65, F=46)$} & \multirow[t]{2}{*}{$t$} & \multirow[t]{2}{*}{$p$} \\
\hline & M & SD & M & SD & & \\
\hline Age & 33.3 & 9.56 & 33.3 & 10.2 & 0.004 & 1.00 \\
\hline Rectal discomfort threshold (mmHg) & 24.4 & 7.1 & 21.2 & 7.8 & 1.66 & 0.10 \\
\hline Rectal pain threshold (mmHg) & 30.5 & 10.3 & 27.4 & 8.8 & 1.33 & 0.19 \\
\hline First rectal sensation (mmHg) & 10.3 & 5.1 & 9.9 & 5.9 & 0.23 & 0.82 \\
\hline IBS-SSS & 26 & 31.3 & 280 & 100 & -11.36 & $1.15 \times 10^{-18}$ \\
\hline Rectal discomfort intensity (VAS, mm) & 49.8 & 22.1 & 68.7 & 25.7 & -3.05 & 0.003 \\
\hline Rectal pain intensity (VAS, mm) & 25.4 & 31.33 & 40.4 & 31.5 & -2.4 & 0.019 \\
\hline Lactulose challenge-pain (AUC) & 60.7 & 166.41 & 1139.4 & 1243.07 & -3.95 & 0.0002 \\
\hline Lactulose challenge-discomfort (AUC) & 212.5 & 227.9 & 1911.1 & 1062.2 & -7.23 & $1.91 \times 10^{-10}$ \\
\hline OATT (days) & 1.5 & 0.7 & 1.6 & 1.2 & -0.26 & 0.84 \\
\hline
\end{tabular}




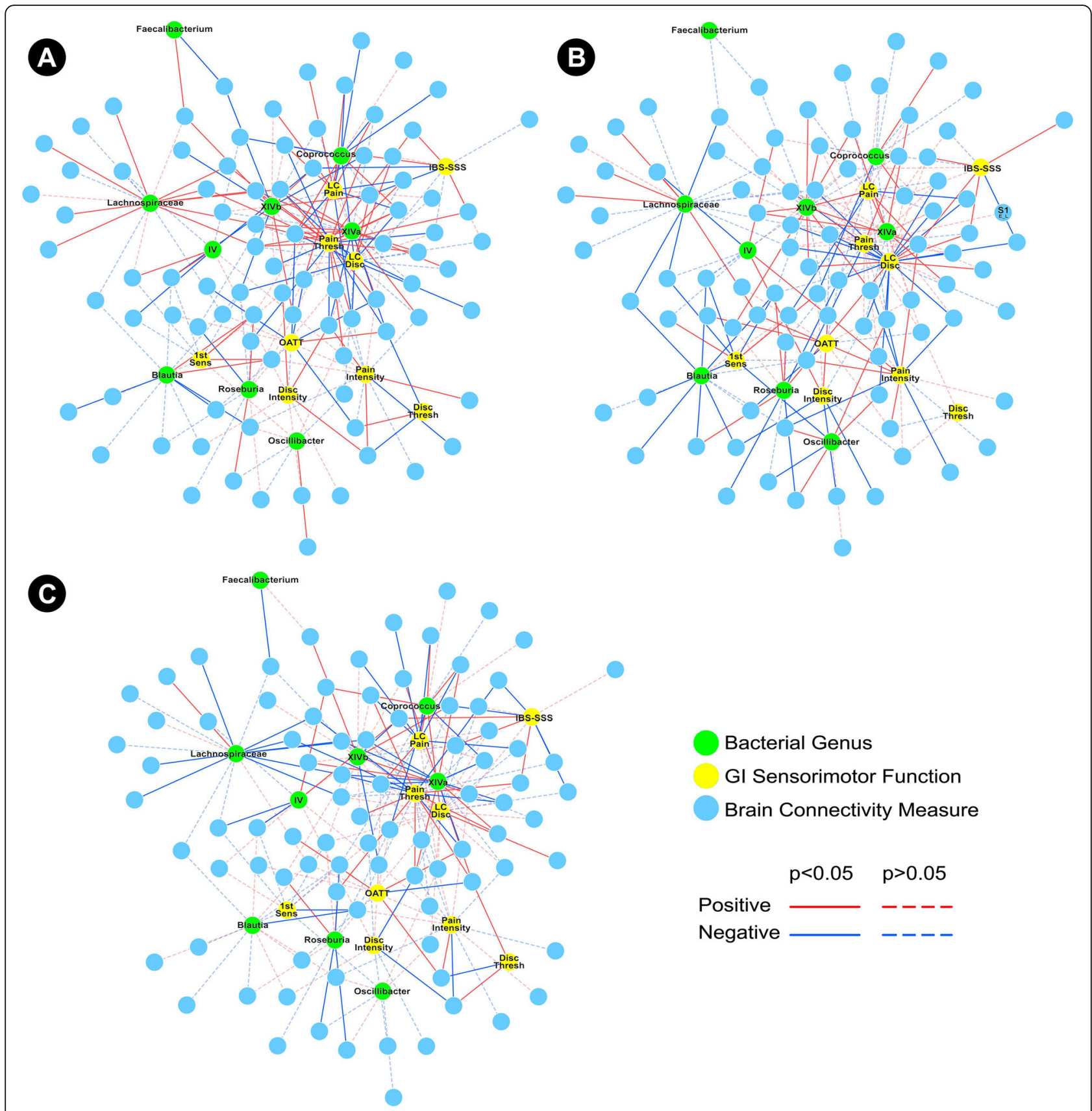

Fig. 1 Global tripartite microbial genus-brain connectivity-evoked symptom network. a Demonstrates the global tripartite healthy controls network. $\mathbf{b}$ Demonstrates the global tripartite IBS network. c Demonstrates the global tripartite difference network. For clarity, labels related to brain connectivity measures have been omitted from these global networks. 1st Sens first sensation threshold during balloon distension, Disc Thresh discomfort threshold during balloon distension, IBS-SS irritable bowel syndrome-scoring system scores, IV Clostridium IV, LC Disc discomfort during lactulose challenge test, LC Pain pain during lactulose challenge test, OATT oroanal transit time, Pain Thresh pain threshold during balloon distension, Disc Intensity visual analogue scale rating of discomfort during $24 \mathrm{mmHg}$ distension, Pain Intensity visual analogue scale rating of pain during $24 \mathrm{mmHg}$ distension, IBS-SSS IBS Severity Scoring System, XIVa Clostridium XIVa, XIVb Clostridium XIVb, Lachnospiraceae Lachnospiraceae incertae sedis

was also a part of the Clostridium XIVb subnetwork, also showed an indirect association with IBS-SSS through connectivity of the same region of M1 (Fig. 5a). In contrast, the IBS subnetwork did not demonstrate any associations between Clostridium
$X I V b$ and IBS-SSS, but did show a positive association between this genus and connectivity of the thalamus (Fig. 5b). The Clostridium XIVb difference network underscores that the observed differences are significant (Fig. 5c). 


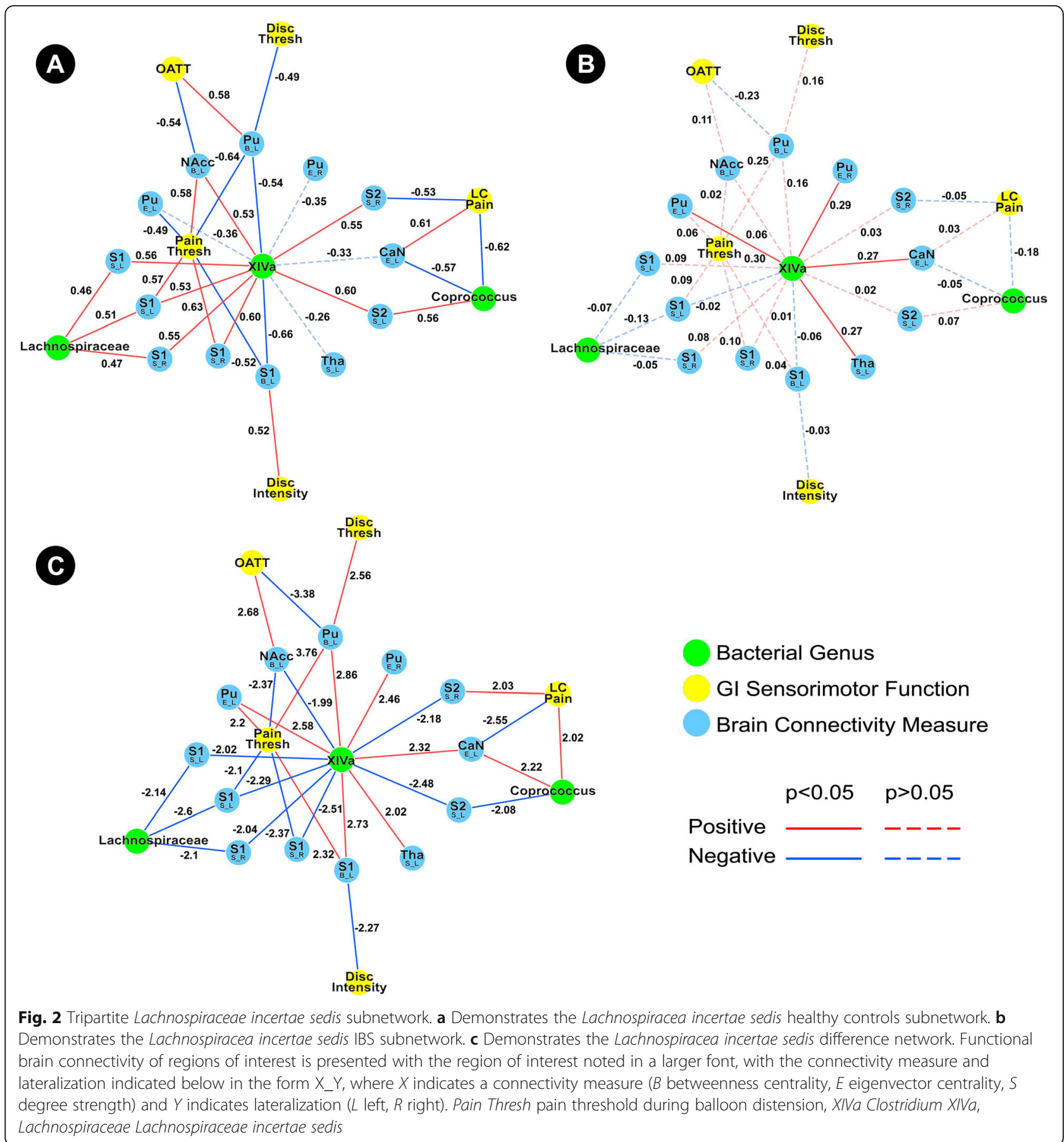

\section{Discussion}

In this study, we demonstrate differences in microbial subnetworks of IBS patients and HCs that may reflect alterations in the interactions within the brain-gut-microbiome axis of IBS subjects. Our tripartite analysis both supports previous neuroimaging studies in this patient population, and also demonstrates novel findings that may provide the foundation for future, larger, mechanistic studies. As we have previously shown in preclinical studies that members of the order Clostridiales (particularly enriched with families Ruminococcaceae and Lachnospiraceae) stimulate the biosynthesis and release of 5-HT from intestinal enterochromaffin cells and modulate GI motility, we focused exclusively on genera from this subset of microbes [12]. Although these results were obtained in a relatively small study population, and will need to be confirmed in a much larger study, to our knowledge, this is the first study demonstrating differences between microbial genera 


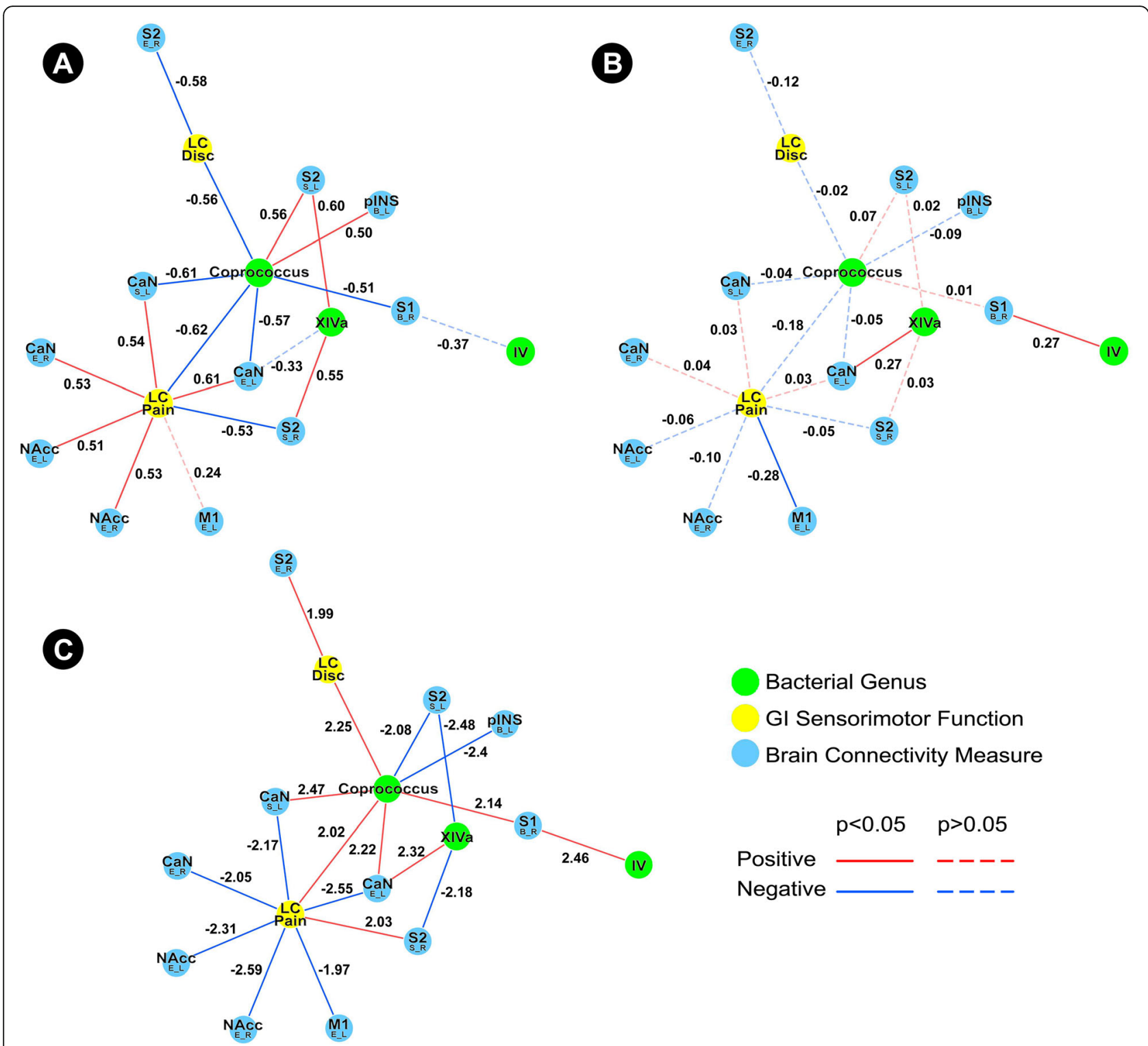

Fig. 3 Tripartite Coprococcus Subnetwork. a Demonstrates the Coprococcus healthy controls subnetwork. b Demonstrates the Coprococcus IBS subnetwork. c Demonstrates the Coprococcus difference network. Functional brain connectivity of regions of interest is presented with the region of interest noted in a larger font, with the connectivity measure and lateralization indicated below in the form X_Y, where $X$ indicates a connectivity measure ( $B$ betweenness centrality, $E$ eigenvector centrality, $S$ degree strength) and $Y$ indicates lateralization ( $L$ left, $R$ right). IV Clostridium IV, LC Disc discomfort during lactulose challenge test, LC Pain pain during nutrient and lactulose challenge test, XIVa Clostridium XIVa

subnetworks in IBS patients and HCs with respect to functional connectivity of brain regions in the somatosensory network and GI sensorimotor function.

\section{Tripartite analysis reveals disruptions in IBS network} architecture

Overall, the HC subnetworks demonstrate numerous associations with GI sensorimotor function, which were absent from the IBS group. In HCs, the subnetworks of Lachnospiraceae incertae sedis, Clostridium XIVa, and Coprococcus, in particular, demonstrate robust interactions between their respective microbial genera and GI sensorimotor function through connectivity of regions within the somatosensory network. This is in contrast to the less robust subnetworks in IBS subjects, which are characterized by a loss of these, perhaps protective, associations.

In HCs, the Lachnospiraceae incertae sedis subnetwork demonstrates indirect associations between Lachnospiraceae incertae sedis and rectal pain threshold through connectivity of S1. In contrast, the IBS subnetwork is significantly less robust, showing no associations, direct 


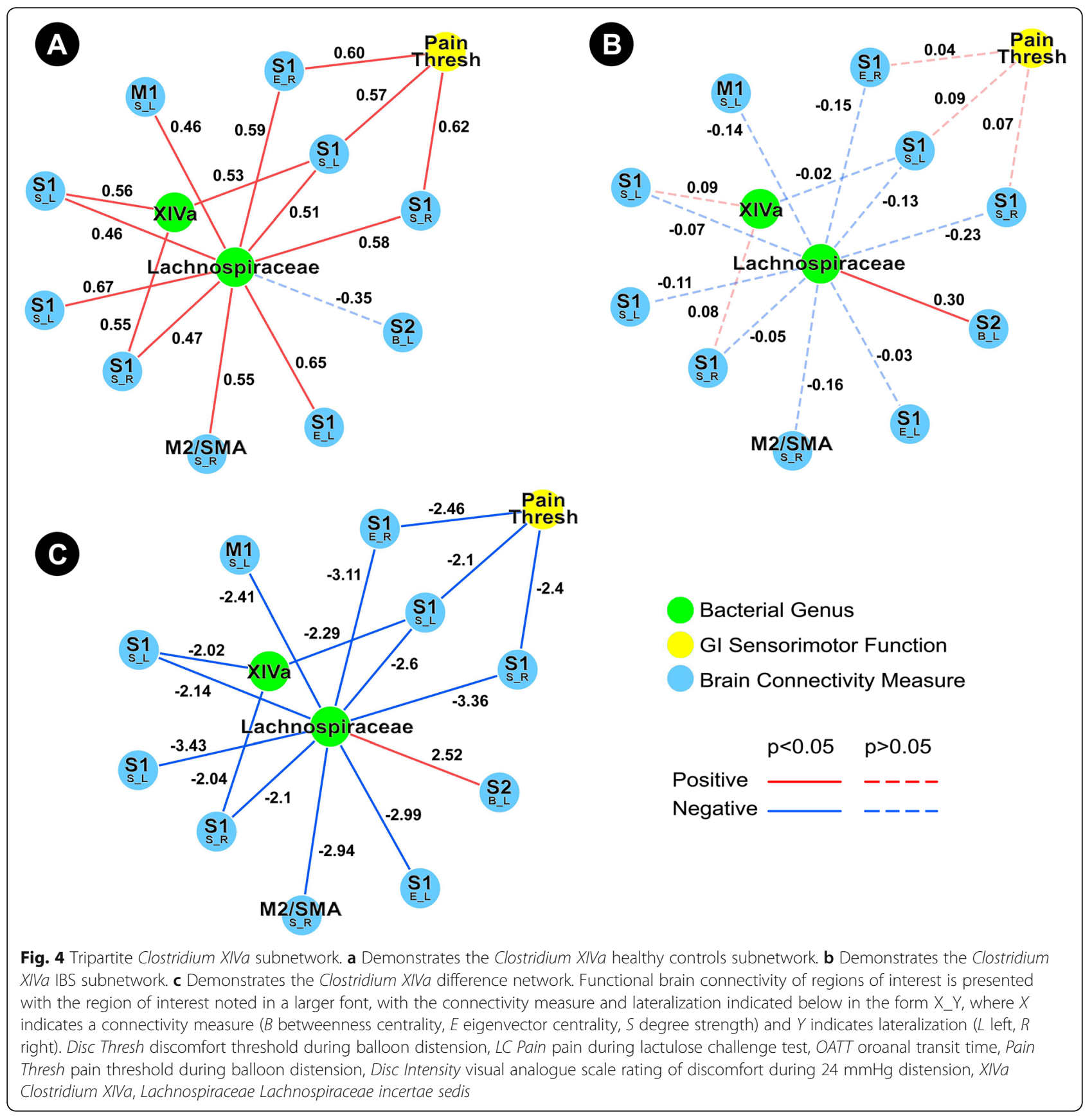

or indirect, between this genus and any measure of GI sensorimotor function. However, in contrast to the numerous positive associations between Lachnospiraceae incertae sedis and S1 in HCs, the IBS subnetwork showed a positive association between this genus and connectivity of S2. Though both S1 and S2 are involved in processing painful sensorimotor input, $\mathrm{S} 1$ has been documented to play an important role in the localization and discrimination of painful and noxious stimuli intensity, whereas S2 performs higher order functions involving sensorimotor integration, attention, learning, and memory related to pain [39-41]. The differences in Lachnospiraceae incertae sedis subnetworks between the $\mathrm{HCs}$ and IBS reported here are supported by previous studies that show altered activity in S1 and S2, as well as an association of these alterations with visceral sensitivity in fMRI studies of IBS patients [42]. Although no conclusions about mechanisms underlying the observed differences in the Lachnospiraceae incertae sedis subnetworks (or subnetworks involving other genera) can be made from the current cross sectional study, a plausible explanation involves a disruption in this genus' ability to 


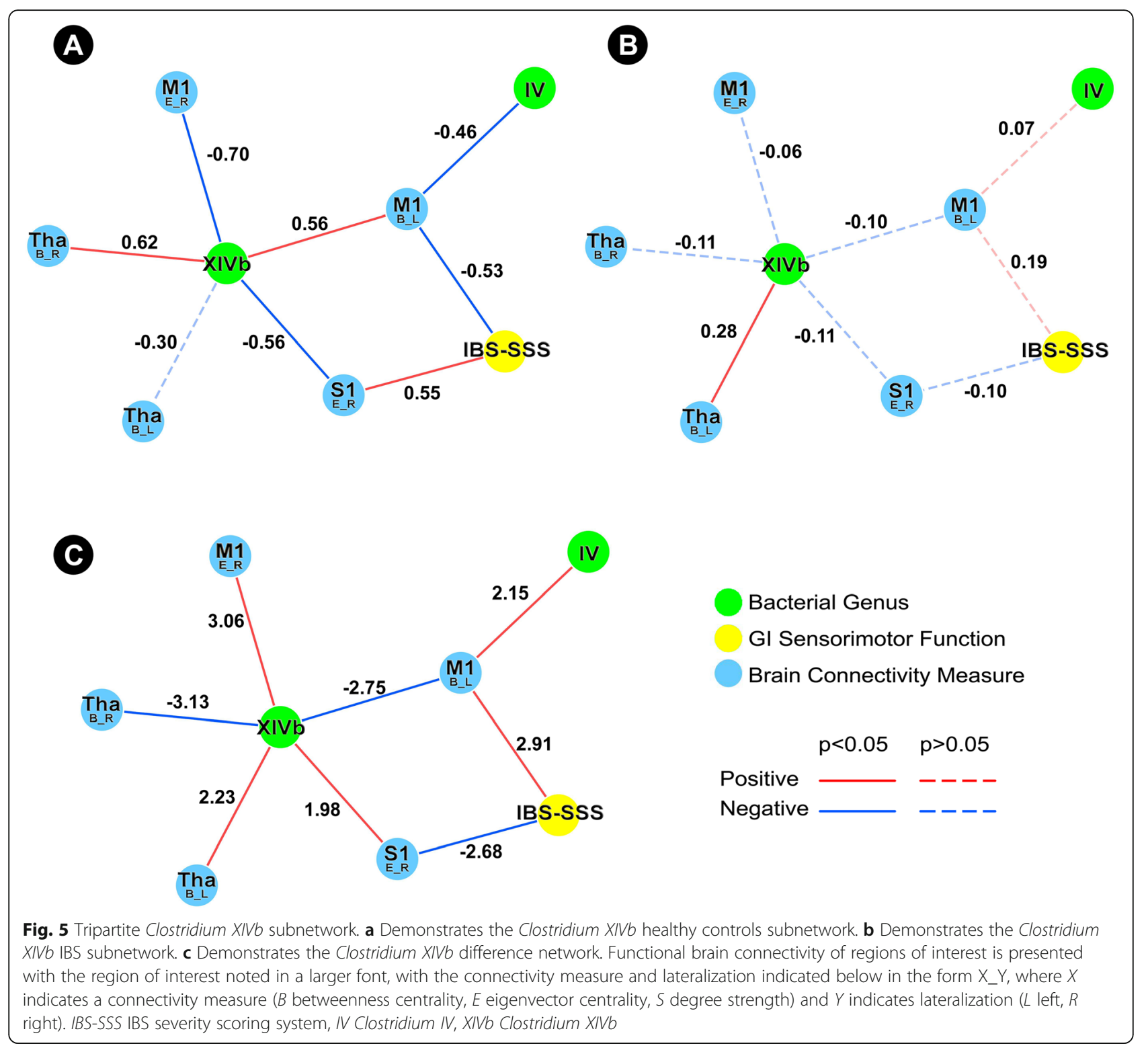

modulate the host serotonergic system. In support of this, one study found that Lachnospiraceae incertae sedis was increased in patients with diarrhea-predominant IBS, a subgroup that has been associated with increased plasma 5-HT levels [43, 44].

Similar to the Lachnospiraceae incertae sedis subnetworks, the Clostridium XIVa and Coprococcus subnetworks show numerous indirect associations between these genera and GI sensorimotor function in $\mathrm{HCs}$, but not in IBS. Many of these indirect associations involve connectivity of subcortical brain regions. More specifically, the Clostridium XIVa HC subnetwork demonstrates indirect associations between Clostridium XIVa with rectal pain threshold (through connectivity of the putamen, NAcc, and S1), rectal discomfort threshold (through connectivity of the putamen), rectal discomfort intensity (through connectivity of S1), pain during the nutrient and lactulose challenge test (through connectivity of S2), and OATT (through connectivity of the putamen and NAcc). The Coprococcus $\mathrm{HC}$ subnetwork demonstrates direct negative associations between Coprococcus and pain and discomfort during the nutrient and lactulose challenge test, in addition to indirect associations between Coprococcus and pain during this challenge test through connectivity of the caudate. In contrast, the IBS subnetworks are more sparse, with the Coprococcus subnetwork showing no meaningful associations. The Clostridium XIVa subnetwork does demonstrate exclusively positive associations between Clostridium XIVa and connectivity of subcortical regions (putamen, caudate, and thalamus).

These results align with a previous report from our group, which showed positive associations between 
Firmicutes-associated Clostridia (such as Coprococcus and Clostridium XIVa) and increased gray matter volume of the putamen, caudate, and nucleus accumbens in IBS patients [11]. Numerous studies have demonstrated activation of subcortical regions, in particular the putamen, during both acute and chronic pain $[45,46]$. Additionally, previous fMRI studies involving experimental rectal stimulation have demonstrated a role for the basal ganglia in IBS-specific alterations in pain processing [4, 47]. One study identified microstructural reorganization in basal ganglia regions in patients with IBS [48]. Deep brain stimulation of the caudate nucleus in a patient with obsessive compulsive disorder and IBS reduced both psychiatric and gastrointestinal symptoms [49]. Coprococcus and Clostridium XIVa may contribute to visceral hypersensitivity and pain in IBS by influencing these subcortical regions.

In HCs, Clostridium XIVb and Clostridium IV showed indirect associations with IBS-SSS through connectivity of cortical regions (M1 and S1). In IBS, although Clostridium XIVb showed an indirect association with IBS-SSS through connectivity of the thalamus, this association did not emerge as significant with respect to the difference network. Interestingly, IBS-SSS did not show more numerous associations with microbial genera in the IBS subnetworks. A previous study, which identified an intestinal microbiota signature associated with severity of IBS, suggested an important role for operational taxonomic units/"species" in the genera Roseburia, Lachnospiraceae incertae sedis, and Clostridium XIVa in IBS symptom severity [10]. The difference in our results from this previous study could be attributed to differences in methodology and study design, as the aforementioned study used machine learning approaches and operational taxonomic units, rather than genera.

\section{Limitations}

This study focused on microorganisms and brain regions that have previously been implicated in IBS pathophysiology. The specific microorganisms have been shown in preclinical studies to modulate host serotonin biosynthesis and release in the GI tract and to be associated with elevated relative abundances in subsets of patients with IBS $[11,12]$. Future studies in larger populations may benefit from expanding the genera we have investigated, or alternatively, exploring the effects of individual species or operational taxonomic units. Due to the limited sample size, we did not investigate the influence of psychiatric variables (anxiety, depression) or history of early adversity that may confound the findings presented in this study. Due to the small sample size and the exploratory nature of this study, correction for multiple comparisons was not performed. Alternative analytical approaches to data analysis, perhaps involving methods specific for operational taxonomical unit (OTU)-OTU correlation networks rather than for abundance data correlation networks, should be explored in future work. Although future, larger studies of this type may reveal differences in certain components of the subnetworks we have presented here, it is likely that the overall patterns and differences in subnetworks between IBS and HCs will remain. As the microorganisms investigated in this study have been implicated in serotonergic modulation, future studies should incorporate measurements of 5-HT and other tryptophan metabolites in their investigation of BGM interactions in IBS. As this is an association study, no causative relationships can be implied, and the directionality of the interactions between relative microbial abundance and brain connectivity cannot be parsed out. However, previous work has suggested a bidirectional model for brain-gut-microbiome communication, with evidence for both top-down and bottom-up communication as playing an important role in shaping the IBS and HCs subnetworks we have demonstrated. [50]

\section{Conclusions}

In this study, we build on our previous work on brain gut microbiome interactions that has suggested a role for microorganisms in the order Clostridiales in modulating host 5-HT biosynthesis and release, and influencing brain regions in patients with IBS. To our knowledge, this is the first study investigating differences between IBS and HCs with respect to the interaction network between microorganisms, functional connectivity of brain regions in the somatosensory network, and GI sensorimotor function. Our results suggest that disruptions in the brain-gut-microbiome axis in IBS patients involving mainly subcortical but also cortical brain regions may contribute to visceral hypersensitivity and altered perception of pain in patients with IBS.

\section{Additional files}

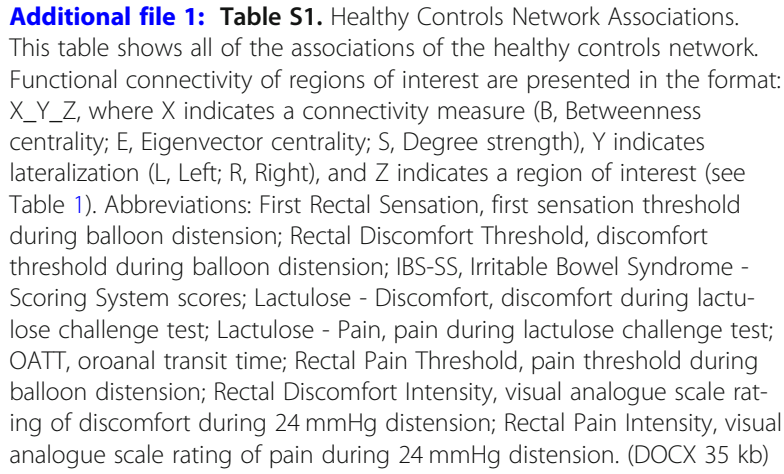

Additional file 1: Table S1. Healthy Controls Network Associations. This table shows all of the associations of the healthy controls network. Functional connectivity of regions of interest are presented in the format: $X \_Y \_Z$, where $X$ indicates a connectivity measure $(B$, Betweenness centrality; E, Eigenvector centrality; $S$, Degree strength), $Y$ indicates lateralization ( $L$, Left; $R$, Right), and $Z$ indicates a region of interest (see Table 1). Abbreviations: First Rectal Sensation, first sensation threshold during balloon distension; Rectal Discomfort Threshold, discomfort threshold during balloon distension; IBS-SS, Irritable Bowel Syndrome Scoring System scores; Lactulose - Discomfort, discomfort during lactulose challenge test; Lactulose - Pain, pain during lactulose challenge test; OATT, oroanal transit time; Rectal Pain Threshold, pain threshold during balloon distension; Rectal Discomfort Intensity, visual analogue scale rating of discomfort during $24 \mathrm{mmHg}$ distension; Rectal Pain Intensity, visual analogue scale rating of pain during $24 \mathrm{mmHg}$ distension. (DOCX $35 \mathrm{~kb}$ )

Additional file 2: Table S2. IBS Network Associations. This table shows all of the associations of the IBS network. Functional connectivity of regions of interest are presented in the format: $X_{-} Y_{-} Z$, where $X$ indicates 
a connectivity measure $\left(B\right.$, Betweenness centrality; $E_{\text {, Eigenvector }}$ centrality; $S$, Degree strength), $Y$ indicates lateralization ( $L$, Left; $R$, Right) and $Z$ indicates a region of interest (see Table 1). Abbreviations: First Rectal Sensation, first sensation threshold during balloon distension; Rectal Discomfort Threshold, discomfort threshold during balloon distension; IBS-SS, Irritable Bowel Syndrome - Scoring System scores; Lactulose - Discomfort, discomfort during lactulose challenge test; Lactulose - Pain, pain during nutrient and lactulose challenge test; OATT, oroanal transit time; Rectal Pain Threshold, pain threshold during balloon distension; Rectal Discomfort Intensity, visual analogue scale rating of discomfort during $24 \mathrm{mmHg}$ distension; Rectal Pain Intensity, visual analogue scale rating of pain during $24 \mathrm{mmHg}$ distension (DOCX $150 \mathrm{~kb}$ )

Additional file 3: Table S3. Difference Network Z Tests. This table shows all of the $Z$ tests that define the difference network. Functional connectivity of regions of interest are presented in the format: $X_{-} Y{ }_{-} Z_{\text {, }}$ where $\mathrm{X}$ indicates a connectivity measure (B, Betweenness centrality; $E$, Eigenvector centrality; S, Degree strength), $Y$ indicates lateralization $(L$, Left; $R$, Right), and $Z$ indicates a region of interest (see Table 1). Abbreviations: First Rectal Sensation, first sensation threshold during balloon distension; Rectal Discomfort Threshold, discomfort threshold during balloon distension; IBS-SS, Irritable Bowel Syndrome - Scoring System scores; Lactulose - Discomfort, discomfort during nutrient and lactulose challenge test; Lactulose - Pain, pain during lactulose challenge test; OATT, oroanal transit time; Rectal Pain Threshold, pain threshold during balloon distension; Rectal Discomfort Intensity, visual analogue scale rating of discomfort during $24 \mathrm{mmHg}$ distension; Rectal Pain Intensity, visual analogue scale rating of pain during $24 \mathrm{mmHg}$ distension. DOCX $156 \mathrm{~kb})$

Additional file 4: Supplemental Materials. Supplemental materials related to statistical power calculations. (DOCX $71 \mathrm{~kb}$ )

\section{Abbreviations}

5-HT: Serotonin; CNS: Central nervous system; FODMAP: Fermentable oligosaccharides, disaccharides, monosaccharides, and polyols; GI: Gastrointestinal; HC: Healthy control; IBS: Irritable bowel syndrome; IBSSSS: IBS severity scoring system; NAcc: Nucleus accumbens

\section{Acknowledgements}

We would like to acknowledge the invaluable assistance of Cathy Liu.

\section{Funding}

This study was supported by grants from the National Institute of Diabetes and Digestive and Kidney Diseases (DK048351, DK064539 and DK096606), and by the Swedish Medical Research Council (grants 13409, 21691 and 21692), AFA Insurance, an unrestricted grant from Danone Nutricia Research, and the Faculty of Medicine, University of Gothenburg.

\section{Availability of data and materials}

The datasets used and analyzed during the current study and from the parent study [10] are available at: https://doi.org/10.5878/ejpj-p674, https:// doi.org/10.5878/5rbz-ww62.

\section{Authors' contributions}

JSL and VO: study concept and design, analysis and interpretation of data, drafting of the manuscript, critical revision of the manuscript for important intellectual content, and statistical analysis. EYH: interpretation of data and drafting of the manuscript. AG: statistical analysis, technical support. KT: critical revision of the manuscript for important intellectual content. BLN, CG, LO, JT, MD: critical revision of the manuscript for important intellectual content. ML: technical support, critical revision of the manuscript for important intellectual content. HT: acquisition of data, critical revision of the manuscript for important intellectual content. MS: funding, study concept and design, critical revision of the manuscript for important intellectual content. EAM: funding, study concept and design, critical revision of the manuscript for important intellectual content, study supervision. All authors read and approved the final manuscript.

\section{Ethics approval and consent to participate}

All subjects were given verbal and written information about the study prior to providing written informed consent to participate. The Regional Ethical
Review Board in Gothenburg approved the study before the start of subject inclusion.

\section{Consent for publication}

Not applicable.

\section{Competing interests}

JSL, VO, EYH, AG, KT, CG, ML, LO, and HT have nothing to declare. BLN, MD, and JT are employees of Danone Nutricia Research. MS received unrestricted research grants from Danone Nutricia Research and Ferring Pharmaceuticals, and served as a Consultant/Advisory Board member for AstraZeneca, Danone, Nestlé, Almirall, Allergan, Albireo, Glycom, and Shire, and as a speaker for Tillotts, Menarini, Takeda, Shire, Allergan, and Almirall. EAM is a scientific advisory board member of Dannon, Danone, DanoneWave, Axial Biotherapeutics, Viome, Amare, and Pharmavite.

\section{Publisher's Note}

Springer Nature remains neutral with regard to jurisdictional claims in published maps and institutional affiliations.

\section{Author details}

${ }^{1}$ G. Oppenheimer Center for Neurobiology of Stress \& Resilience, UCLA Vatche and Tamar Manoukian Division of Digestive Diseases, UCLA CHS 42-210, MC737818, 10833 Le Conte Avenue, Los Angeles, CA 90095-7378, USA. ${ }^{2}$ UCLA Department of Integrative Biology and Physiology, Los Angeles, USA. ${ }^{3}$ Danone Nutricia Research, Innovation, Science and Nutrition, Palaiseau, France. ${ }^{4}$ Department of Internal Medicine and Clinical Nutrition, Institute of Medicine, Sahlgrenska Academy, University of Gothenburg, Gothenburg, Sweden. ${ }^{5}$ Department of Radiation Physics, Institute of Clinical Sciences, Sahlgrenska Academy, University of Gothenburg, Gothenburg, Sweden. ${ }^{6}$ Department of Immunology and Microbiology, Institute of Biomedicine, Sahlgrenska Academy, University of Gothenburg, Gothenburg, Sweden. ${ }^{7}$ Center for Functional Gastrointestinal and Motility Disorders, University of North Carolina, Chapel Hill, NC, USA.

Received: 1 October 2018 Accepted: 7 March 2019

Published online: 21 March 2019

\section{References}

1. Mayer EA, Savidge T, Shulman RJ. Brain-gut microbiome interactions and functional bowel disorders. Gastroenterology. 2014;146:1500-12.

2. Mayer EA, Gupta A, Kilpatrick LA, Hong JY. Imaging brain mechanisms in chronic visceral pain. Pain. 2015:156(Suppl 1):S50-63.

3. Labus JS, Van Horn JD, Gupta A, Alaverdyan M, Torgerson C, Ashe-McNalley C, Irimia A, Hong JY, Naliboff B, Tillisch K, Mayer EA. Multivariate morphological brain signatures predict patients with chronic abdominal pain from healthy control subjects. Pain. 2015;156:1545-54.

4. Tillisch K, Mayer EA, Labus JS. Quantitative meta-analysis identifies brain regions activated during rectal distension in irritable bowel syndrome. Gastroenterology. 2011;140:91-100.

5. Simren M, Tornblom H, Palsson OS, van Tilburg MAL, Van Oudenhove L, Tack J, Whitehead WE. Visceral hypersensitivity is associated with Gl symptom severity in functional Gl disorders: consistent findings from five different patient cohorts. Gut. 2018:67:255-62

6. Posserud I, Syrous A, Lindstrom L, Tack J, Abrahamsson H, Simren M. Altered rectal perception in irritable bowel syndrome is associated with symptom severity. Gastroenterology. 2007;133:1113-23.

7. Tornblom H, Van Oudenhove L, Sadik R, Abrahamsson H, Tack J, Simren M. Colonic transit time and IBS symptoms: what's the link? Am J Gastroenterol. 2012;107:754-60.

8. Collins SM. A role for the gut microbiota in IBS. Nat Rev Gastroenterol Hepatol. 2014;11:497-505.

9. Ohman L, Simren M. Intestinal microbiota and its role in irritable bowel syndrome (IBS). Curr Gastroenterol Rep. 2013;15:323.

10. Tap J, Derrien M, Tornblom H, Brazeilles R, Cools-Portier S, Dore J, Storsrud S, Le Neve B, Ohman L, Simren M. Identification of an intestinal microbiota signature associated with severity of irritable bowel syndrome. Gastroenterology. 2017;152:111-123 e118.

11. Labus JS, Hollister EB, Jacobs J, Kirbach K, Oezguen N, Gupta A, Acosta J, Luna RA, Aagaard K, Versalovic J, et al. Differences in gut microbial 
composition correlate with regional brain volumes in irritable bowel syndrome. Microbiome. 2017:5:49.

12. Yano JM, Yu K, Donaldson GP, Shastri GG, Ann P, Ma L, Nagler CR, Ismagilov RF, Mazmanian SK, Hsiao EY. Indigenous bacteria from the gut microbiota regulate host serotonin biosynthesis. Cell. 2015;161:264-76.

13. Mawe GM, Hoffman JM. Serotonin signalling in the gut--functions, dysfunctions and therapeutic targets. Nat Rev Gastroenterol Hepatol. 2013, 10:473-86.

14. Sikander A, Rana SV, Prasad KK. Role of serotonin in gastrointestinal motility and irritable bowel syndrome. Clin Chim Acta. 2009;403:47-55.

15. Houghton LA, Atkinson W, Lockhart C, Whorwell PJ, Keevil B. Sigmoidcolonic motility in health and irritable bowel syndrome: a role for 5hydroxytryptamine. Neurogastroenterol Motil. 2007;19:724-31.

16. Houghton LA, Atkinson W, Whitaker RP, Whorwell PJ, Rimmer MJ. Increased platelet depleted plasma 5-hydroxytryptamine concentration following meal ingestion in symptomatic female subjects with diarrhoea predominant irritable bowel syndrome. Gut. 2003:52:663-70

17. Martin CR, Osadchiy V, Kalani A, Mayer EA. The brain-gut-microbiome axis. Cell Mol Gastroenterol Hepatol. 2018;6:133-48.

18. Halmos EP, Power VA, Shepherd SJ, Gibson PR, Muir JG. A diet low in FODMAPs reduces symptoms of irritable bowel syndrome. Gastroenterology. 2014;146:67-75 e65.

19. Altobelli E, Del Negro V, Angeletti PM, Latella G. Low-FODMAP diet improves irritable bowel syndrome symptoms: a meta-analysis. Nutrients. 2017;9

20. Carabotti M, Scirocco A, Maselli MA, Severi C. The gut-brain axis: interactions between enteric microbiota, central and enteric nervous systems. Ann Gastroenterol. 2015;28:203-9.

21. Osadchiy V, Labus JS, Gupta A, Jacobs J, Ashe-McNalley C, Hsiao EY, Mayer EA. Correlation of tryptophan metabolites with connectivity of extended central reward network in healthy subjects. PLoS One. 2018;13:e0201772.

22. Sporns O. From simple graphs to the connectome: networks in neuroimaging. Neuroimage. 2012;62:881-6.

23. Bullmore $\mathrm{E}$, Sporns $\mathrm{O}$. Complex brain networks: graph theoretical analysis of structural and functional systems. Nat Rev Neurosci. 2009;10:186-98.

24. Irimia A, Chambers MC, Torgerson CM, Van Horn JD. Circular representation of human cortical networks for subject and population-level connectomic visualization. Neuroimage. 2012;60:1340-51.

25. Rubinov M, Sporns O. Complex network measures of brain connectivity: uses and interpretations. Neuroimage. 2010;52:1059-69.

26. Longstreth GF, Thompson WG, Chey WD, Houghton LA, Mearin F, Spiller RC. Functional bowel disorders. Gastroenterology. 2006;130:1480-91.

27. Jeffery IB, O'Toole PW, Ohman L, Claesson MJ, Deane J, Quigley EM, Simren M. An irritable bowel syndrome subtype defined by species-specific alterations in faecal microbiota. Gut. 2012;61:997-1006.

28. Francis CY, Morris J, Whorwell PJ. The irritable bowel severity scoring system: a simple method of monitoring irritable bowel syndrome and its progress. Aliment Pharmacol Ther. 1997;11:395-402.

29. Cremonini F, Houghton LA, Camilleri M, Ferber I, Fell C, Cox V, Castillo EJ, Alpers DH, Dewit OE, Gray E, et al. Barostat testing of rectal sensation and compliance in humans: comparison of results across two centres and overall reproducibility. Neurogastroenterol Motil. 2005;17:810-20.

30. Le Neve B, Posserud I, Bohn L, Guyonnet D, Rondeau P, Tillisch K, Naliboff B, Mayer EA, Simren M. A combined nutrient and lactulose challenge test allows symptom-based clustering of patients with irritable bowel syndrome. Am J Gastroenterol. 2013;108:786-95.

31. Matsuki T, Watanabe K, Fujimoto J, Takada T, Tanaka R. Use of $16 \mathrm{~S}$ rRNA gene-targeted group-specific primers for real-time PCR analysis of predominant bacteria in human feces. Appl Environ Microbiol. 2004;70: 7220-8.

32. Godon JJ, Zumstein E, Dabert P, Habouzit F, Moletta R. Molecular microbial diversity of an anaerobic digestor as determined by small-subunit rDNA sequence analysis. Appl Environ Microbiol. 1997;63:2802-13

33. Whitfield-Gabrieli S, Nieto-Castanon A. Conn: a functional connectivity toolbox for correlated and anticorrelated brain networks. Brain Connect. 2012:2:125-41.

34. Spielberg JM, Miller GA, Heller W, Banich MT. Flexible brain network reconfiguration supporting inhibitory control. Proc Natl Acad Sci U S A. 2015;112:10020-5.

35. Icenhour A, Witt ST, Elsenbruch S, Lowen M, Engstrom M, Tillisch K, Mayer EA, Walter S. Brain functional connectivity is associated with visceral sensitivity in women with irritable bowel syndrome. Neuroimage Clin. 2017; 15:449-57.

36. Hong JY, Naliboff B, Labus JS, Gupta A, Kilpatrick LA, Ashe-McNalley C, Stains J, Heendeniya N, Smith SR, Tillisch K, Mayer EA. Altered brain responses in subjects with irritable bowel syndrome during cued and uncued pain expectation. Neurogastroenterol Motil. 2016;28:127-38.

37. Mayer EA, Labus JS, Tillisch K, Cole SW, Baldi P. Towards a systems view of IBS. Nat Rev Gastroenterol Hepatol. 2015;12:592-605.

38. Cohen J. Statistical power analysis for the behavioral sciences. 2. Edn. Hillsdale, N.J: Lawrence Erlbaum; 1988.

39. Ogino $Y$, Nemoto H, Goto F. Somatotopy in human primary somatosensory cortex in pain system. Anesthesiology. 2005;103:821-7.

40. Chen TL, Babiloni C, Ferretti A, Perrucci MG, Romani GL, Rossini PM, Tartaro A, Del Gratta C. Human secondary somatosensory cortex is involved in the processing of somatosensory rare stimuli: an fMRI study. Neuroimage. 2008; 40:1765-71.

41. Bushnell MC, Duncan GH, Hofbauer RK, Ha B, Chen Jl, Carrier B. Pain perception: is there a role for primary somatosensory cortex? Proc Natl Acad Sci U S A. 1999;96:7705-9.

42. Mayer EA, Aziz Q, Coen S, Kern M, Labus JS, Lane R, Kuo B, Naliboff B, Tracey I. Brain imaging approaches to the study of functional Gl disorders: a Rome working team report. Neurogastroenterol Motil. 2009;21:579-96.

43. Krogius-Kurikka L, Lyra A, Malinen E, Aarnikunnas J, Tuimala J, Paulin L, Makivuokko H, Kajander K, Palva A. Microbial community analysis reveals high level phylogenetic alterations in the overall gastrointestinal microbiota of diarrhoea-predominant irritable bowel syndrome sufferers. BMC Gastroenterol. 2009;9:95

44. Atkinson W, Lockhart S, Whorwell PJ, Keevil B, Houghton LA. Altered 5hydroxytryptamine signaling in patients with constipation- and diarrheapredominant irritable bowel syndrome. Gastroenterology. 2006;130:34-43.

45. Bingel U, Quante M, Knab R, Bromm B, Weiller C, Buchel C. Subcortical structures involved in pain processing: evidence from single-trial fMRI. Pain. 2002:99:313-21.

46. Chudler EH. Response properties of neurons in the caudate-putamen and globus pallidus to noxious and non-noxious thermal stimulation in anesthetized rats. Brain Res. 1998;812:283-8.

47. Song GH, Venkatraman $\mathrm{V}$, Ho KY, Chee MW, Yeoh KG, Wilder-Smith $\mathrm{CH}$ Cortical effects of anticipation and endogenous modulation of visceral pain assessed by functional brain MRI in irritable bowel syndrome patients and healthy controls. Pain. 2006;126:79-90.

48. Ellingson BM, Mayer E, Harris RJ, Ashe-McNally C, Naliboff BD, Labus JS, Tillisch K. Diffusion tensor imaging detects microstructural reorganization in the brain associated with chronic irritable bowel syndrome. Pain. 2013;154: $1528-41$.

49. Langguth B, Sturm K, Wetter TC, Lange M, Gabriels L, Mayer EA, Schlaier J. Deep brain stimulation for obsessive compulsive disorder reduces symptoms of irritable bowel syndrome in a single patient. Clin Gastroenterol Hepatol. 2015:13:1371-1374 e1373.

50. Mayer EA, Knight R, Mazmanian SK, Cryan JF, Tillisch K. Gut microbes and the brain: paradigm shift in neuroscience. J Neurosci. 2014;34:15490-6.

\section{Ready to submit your research? Choose BMC and benefit from:}

- fast, convenient online submission

- thorough peer review by experienced researchers in your field

- rapid publication on acceptance

- support for research data, including large and complex data types

- gold Open Access which fosters wider collaboration and increased citations

- maximum visibility for your research: over $100 \mathrm{M}$ website views per year

At $\mathrm{BMC}$, research is always in progress.

Learn more biomedcentral.com/submission 